\title{
Measuring the atomic composition of planetary building blocks ${ }^{\star}$
}

\author{
M. K. McClure ${ }^{1,2}$, C. Dominik ${ }^{1}$, and M. Kama ${ }^{3,4}$ \\ 1 Anton Pannekoek Institute for Astronomy, Universiteit van Amsterdam, Science Park 904, 1098 XH Amsterdam, The Netherlands \\ e-mail: mcclure@strw. leidenuniv.nl \\ 2 Leiden Observatory, Leiden University, PO Box 9513, 2300 RA Leiden, The Netherlands \\ 3 Department of Physics and Astronomy, University College London, Gower Street, London WC1E 6BT, UK \\ 4 Tartu Observatory, University of Tartu, Observatooriumi 1, Tõravere 61602, Estonia
}

Received 13 July 2020 / Accepted 10 September 2020

\begin{abstract}
Context. Volatile molecules are critical to terrestrial planetary habitability, yet they are difficult to observe directly where planets form at the midplanes of protoplanetary disks. It is unclear whether the inner $\sim 1 \mathrm{AU}$ of disks are volatile-poor or if this region is resupplied with ice-rich dust from colder disk regions. Dust traps at radial pressure maxima bounding disk gaps can cut off the inner disk from these types of volatile reservoirs. However, the trap retention efficiency and atomic composition of trapped dust have not been measured.

Aims. We present a new technique to measure the absolute atomic abundances in the gas accreting onto T Tauri stars and infer the bulk atomic composition and distribution of midplane solids that have been retained in the disk around the young star TW Hya.

Methods. We identify near-infrared atomic line emission from gas-phase material inside the dust sublimation rim of TW Hya. Gaussian decomposition of the strongest $\mathrm{H}$ Paschen lines isolates the inner disk hydrogen emission. We measure several key elemental abundances, relative to hydrogen, using a chemical photoionization model and infer dust retention in the disk. With a 1D transport model, we determine approximate radial locations and retention efficiencies of dust traps for different elements.

Results. Volatile and refractory elements are depleted from TW Hya's hot gas by factors of $\sim 10^{2}$ and up to $10^{5}$, respectively. The abundances of the trapped solids are consistent with a combination of primitive Solar System bodies. Dust traps beyond the CO and $\mathrm{N}_{2}$ snowline cumulatively sequester $96 \%$ of the total dust flux, while the trap at $2 \mathrm{AU}$, near the $\mathrm{H}_{2} \mathrm{O}$ snowline, retains $3 \%$. The high depletions of $\mathrm{Si}, \mathrm{Mg}$, and $\mathrm{Ca}$ are explained by a third trap at $0.3 \mathrm{AU}$ with $>95 \%$ dust retention.

Conclusion. TW Hya sports a significant volatile reservoir rich in $\mathrm{C}$ - and $\mathrm{N}$-ices in its outer submillimeter ring structure. However, unless the inner disk was enhanced in $\mathrm{C}$ by earlier radial transport, typical $\mathrm{C}$ destruction mechanisms and the lack of a $\mathrm{C}$ resupply should leave the terrestrial planet-forming region of TW Hya "dry" and carbon-poor. Any planets that form within the silicate dust trap at $0.3 \mathrm{AU}$ could resemble Earth in terms of the degree of their volatile depletion.
\end{abstract}

Key words. astrochemistry - techniques: spectroscopic - stars: variables: T Tauri, Herbig Ae/Be - protoplanetary disks

\section{Introduction}

Relative abundances of common elements, such as, C, O, and $\mathrm{Si}$, are used to characterize both planetary atmospheric compositions (Madhusudhan 2012) and those of solar system solid bodies (Bergin et al. 2015). Despite their high solar abundances and enhancement in gas giant atmospheres, $\mathrm{C}, \mathrm{O}$, and $\mathrm{N}$ are depleted relative to $\mathrm{Si}$ on Earth and all classes of meteorites (Bergin et al. 2015; Lodders 2010). The fraction of each element in rocks ("refractories") versus ices ("volatiles") may influence these trends. Ice sublimation depletes solids, and additional mechanisms may exist to destroy $\mathrm{C}$-rich rocky grains in the inner regions of protoplanetary disks (PPDs) while retaining those that are rich in $\mathrm{Si}$ (Anderson et al. 2017). The radial transport of fresh solids from the cold outer disk could renew the $\mathrm{C}$ in the terrestrial planet forming region of PPDs, and it may be that the low $\mathrm{C}$ abundances in Solar System bodies are a consequence of Jupiter segregating the inner solar PPD, rather than a result of destruction by a universal mechanism (Klarmann et al. 2018). However,

* The spectra used in this analysis are only available at the CDS via anonymous ftp to cdsarc. u-strasbg. fr (130.79.128.5) or via http://cdsarc.u-strasbg.fr/viz-bin/cat/J/A+A/642/L15 the midplane distribution of volatile-rich solids in protoplanetary disks has not been observationally well-determined yet due to high optical depths. Moreover, it is unclear where or when these solids start being retained in the disk or "locked" out of the gas accreting onto the central star, which is a key step in the formation of planets (Drake et al. 2005).

The presence of midplane ice reservoirs in the nearby disk TW Hya (Fig. 1) has been inferred from cold gas depletions of $\mathrm{CO}$ and water by factors of $\sim 100$ and $\sim 800$, respectively, relative to the values typically measured for the interstellar medium (ISM; Schwarz et al. 2016; Hogerheijde et al. 2011), depending on the disk chemistry (Kamp et al. 2013). These missing gas species are thought to be located beyond "snowlines", that is to say the locations in PPDs where the midplane temperature is low enough for gas of a given species to freeze out onto dust grains, for example, 4 AU for water and 20 AU for CO in TW Hya (Zhang et al. 2013, 2017). If icy grains grow to at least millimeter sizes, then they no longer vertically circulate into the region where the ice could be photodesorbed by stellar radiation. Dust evolution models predict that these large icy grains will drift radially toward the star faster than the PPD gas, enhancing the gaseous carbon and oxygen abundances inside the CO and water snowlines (Du et al. 2015; 


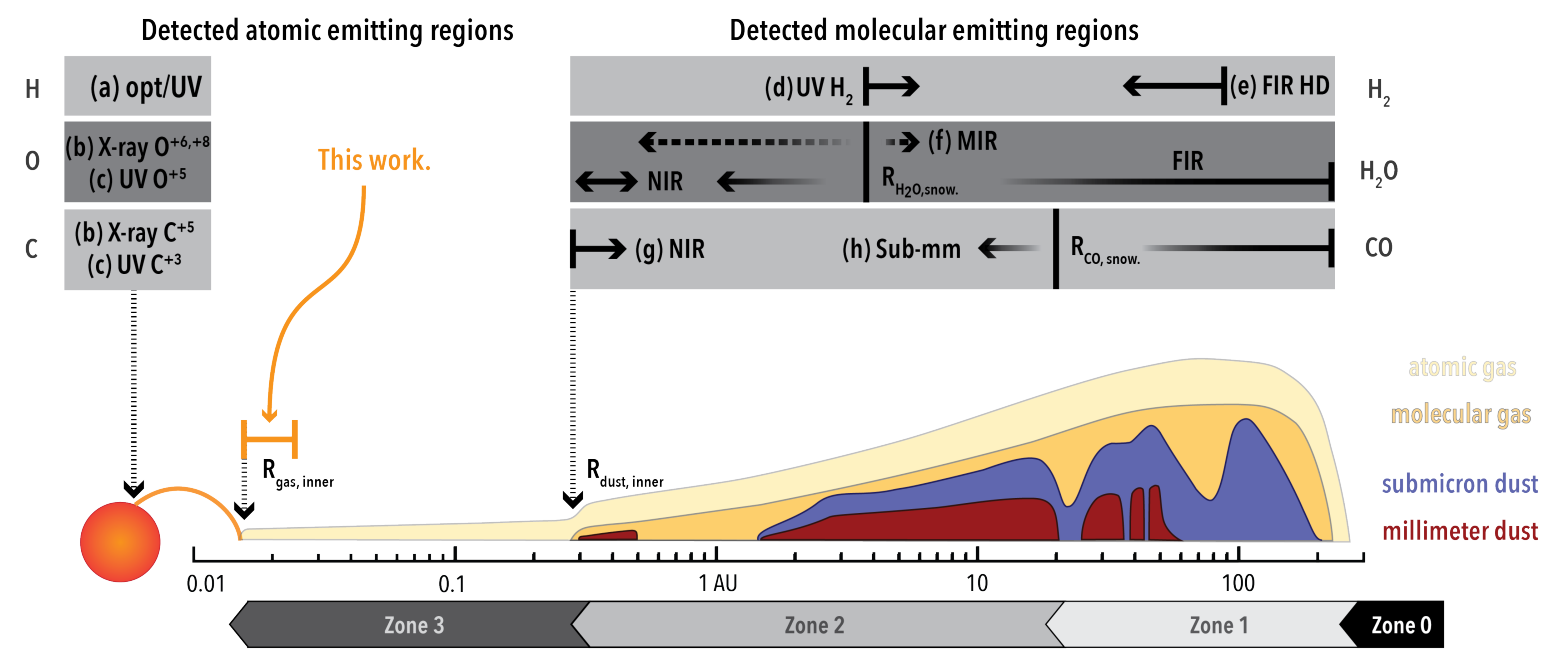

Fig. 1. Schematic of emitting regions for previous TW Hya observations, compared with the region probed in this work. For comparison purposes, observed disk structures in millimeter grains (ALMA, Andrews et al. 2016, VLTI, Menu et al. 2014), micron-sized grains (SPHERE, van Boekel et al. 2017), and CO gas (Banzatti \& Pontoppidan 2015; Huang et al. 2018) are shown. The vertical scale is not meaningful. References: (a) Dupree et al. (2012), (b) Brickhouse et al. (2010), (c) Herczeg et al. (2002), (d) France et al. (2012), (e) Bergin et al. (2013), (f) Zhang et al. (2013), Bosman \& Banzatti (2019), (g) Banzatti \& Pontoppidan (2015), Bosman \& Banzatti (2019), and (h) Zhang et al. (2017). The "zone" labels correspond to the regions described in the analytic calculation of Sect. 4.1.

Krijt et al. 2018), as is seen for HD 163296 (Zhang et al. 2020). In contrast, TW Hya does not show a strong gaseous volatile molecule enhancement inside its $\mathrm{CO}$ snowline at $20 \mathrm{AU}$ (Zhang et al. 2017). Either CO ice is chemically processed into a more rocky carbon carrier or the radial drift of solids is inefficient due to grains collecting in traps (Bosman \& Banzatti 2019), which may be the first step to forming planets or planetesimals themselves.

Bulk gas elemental abundances measured inside the dust sublimation rim can can determine whether the icy $\mathrm{C}$ and $\mathrm{O}$ have returned to the gas phase or are still missing. The former would imply chemical processing of the ice into other ice species with higher sublimation temperatures, while the latter would suggest that the $\mathrm{C}$ and $\mathrm{O}$ have been locked into midplane ice reservoirs. For stars $>1.4 M_{\odot}$, the inner disk elemental abundances can be inferred from the stellar photospheric abundances, which are due to the formation of a radiative outer envelope that isolates recently accreted material at the stellar surface (Kama et al. 2015; Jermyn \& Kama 2018). However, this technique does not work with PPDs around lower mass T Tauri stars, which are the precursors of solar type stars as their outer layers are convective. Interior circulation thus dilutes the compositional signatures of their accreted gas. Recently, we directly measured the inner PPD gas around a set of these lower mass stars through the use of near-infrared atomic C emission lines (McClure 2019). That work inferred carbon locking in these PPDs, but it did not observationally measure abundances of hydrogen or other critical elements, such as $\mathrm{O}$ or $\mathrm{Si}$, which could identify whether these elements were locked in rocky versus icy materials.

In this Letter, we model the hydrogen density and atomic abundances for the inner disk of TW Hya from near-infrared atomic emission lines. We then use these abundances to identify the locations where rock- and ice-forming elements are being locked into midplane dust grain reservoirs in this system.

\section{Data analysis and physical scenario}

To measure the disk abundances inside the dust sublimation radius, we analyzed spectra of TW Hya spanning $0.8-2.5 \mu \mathrm{m}$ taken in three epochs: 2010a and 2010b (VLT X-shooter) as well as 2013 (Magellan FIRE). The observations and data reduction details are given in Appendix A; in brief, the reduced spectra were corrected for telluric absorption, heliocentric velocities, and convolved down to a uniform spectral resolution of $R \sim 6000$. All three epochs show $\mathrm{H}^{0}, \mathrm{He}^{0}, \mathrm{Ca}^{+1}$, and $\mathrm{O}^{0}$ emission lines (Figs. A.1 and A.2). In the 2013 spectrum, these lines were brighter and showed additional emission from $\mathrm{C}^{0}$ and $\mathrm{S}^{0}$ lines at 1.07 and $1.045 \mu \mathrm{m}$, respectively, as is shown in Figs. A.1 and A.2. We subtracted the continuum under the lines of interest in each spectrum using the automated continuum fitter from McClure et al. (2013a). For the detected $\mathrm{Ca}^{+1}, \mathrm{O}^{0}, \mathrm{C}^{0}$, and $\mathrm{S}^{0}$ lines, we integrated the lines and list the fluxes in Table A.1. For the nondetected strong lines, we used the continuum root mean square (rms) value to obtain the $3 \sigma$ upper limits.

The strengthening of all emission lines in 2013 suggests an increase in the density of the accreting, inner disk material for this epoch. We used the $\mathrm{H}$ recombination line profiles to confirm this scenario. As is shown in Fig. 2, the continuumsubtracted Paschen $\gamma$ lines from 2010b and 2013 are both centrally peaked at the stellar rest velocity, with inverse P-Cygni profiles in the wings. However, the maxima and minima of the 2013 profile are stronger. By subtracting the 2010 line profiles from the 2013 spectrum, we removed the contributions of the star and bulk disk to the emission lines. We modeled the residual profile with a four-component Gaussian decomposition. A centrally peaked component is found within the observed velocity resolution uncertainty. Since any stellar chromospheric contribution was removed during the subtraction of the weaker accretion epoch, our fitting results suggest that the centrally peaked component could only come from the inner accretion disk for which the Keplerian motion is in the plane of the sky. In contrast, there is additional inverse P-Cygni emission peaking at $-152 \mathrm{~km} \mathrm{~s}^{-1}$ and absorbing at $+114 \mathrm{~km} \mathrm{~s}^{-1}$. Since TW Hya is face-on (Andrews et al. 2016), we interpret the blueshifted emission component to be the contribution from magnetospheric accretion columns launching out of the disk plane and the red-shifted absorption component to be the post-accretion shock braking region at the stellar surface. A fourth Gaussian 

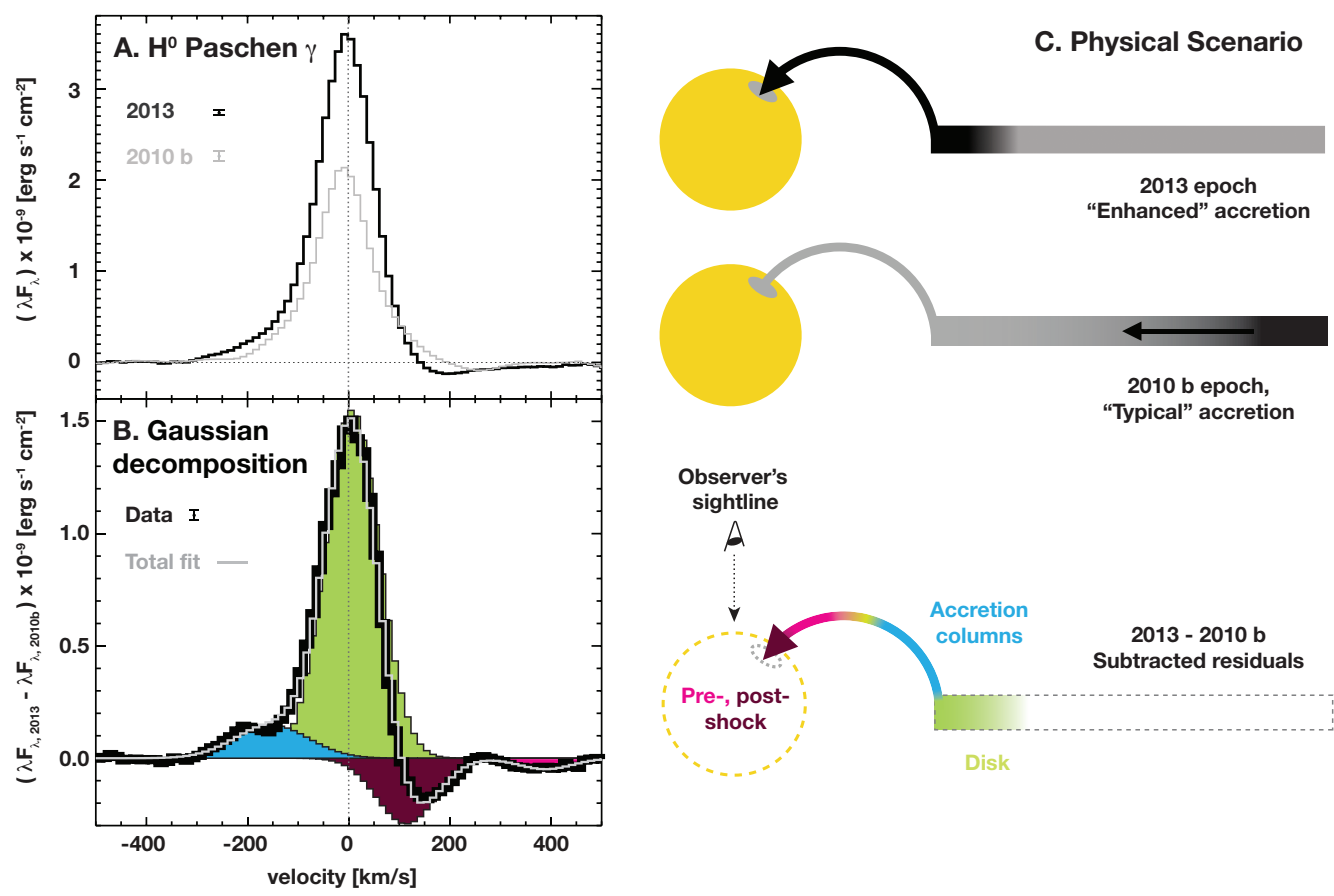

Fig. 2. Isolation of the hydrogen contribution from the inner disk edge. Panel A: comparison of the Pay line emission for the 2010b epoch (gray) and 2013 epoch (black) after continuum subtraction. The dashed vertical line indicates the local stellar velocity to which the spectra were corrected. Panel B: Gaussian decomposition fit (gray) of the residual spectrum (black) obtained by subtracting the 2010b epoch spectrum from that of 2013. The different Gaussian components are indicated by colors and include blue-shifted emission (blue: $-152 \mathrm{~km} \mathrm{~s}^{-1}, F W H M \sim 172 \mathrm{~km} \mathrm{~s}^{-1}$ ), a centrally peaked emission (green: $7 \mathrm{~km} \mathrm{~s}^{-1}, F W H M \sim 126 \mathrm{~km} \mathrm{~s}^{-1}$ ), and two redshifted absorption components (dark red: $114 \mathrm{~km} \mathrm{~s}{ }^{-1}, F W H M \sim$ $137 \mathrm{~km} \mathrm{~s}^{-1}$; bright red: $389 \mathrm{~km} \mathrm{~s}^{-1}, F W H M \sim 124 \mathrm{~km} \mathrm{~s}^{-1}$ ). Panel $C$ : cartoon of the physical scenario revealed by the variability and Gaussian fitting.

component absorbs at $+389 \mathrm{~km} \mathrm{~s}^{-1}$, which is close to the predicted free-fall velocity at the accretion shock interface and $\sim 4$ times the post-shock velocity, as is expected theoretically (Hartmann et al. 2016). Therefore the shape of the $\mathrm{H}$ line profiles support our hypothesis of an increase in the density of the inner disk gas. The physical conditions and elemental abundances in this gas are modeled in the section below.

\section{Modeling}

The Gaussian decomposition fit to the $\mathrm{H}$ line profiles kinematically isolates the 2013 inner disk hydrogen emission. Using an existing 1D model of the inner disks of $\mathrm{T}$ Tauri stars (McClure 2019) driven by the photoionization and chemistry code Cloudy (Ferland et al. 2017), we compared the observed flux ratio between the $\mathrm{H} \mathrm{Pa} \beta$ and $\mathrm{Pa} \gamma$ emission lines to measure, observationally, the $\mathrm{H}$ number density, $n_{\mathrm{H}}$, in the inner disk. Then we modeled the observed flux ratios between these elemental lines and Pa $\gamma$, together with the derived $n_{\mathrm{H}}$, in order to measure total elemental abundances relative to the total amount of $\mathrm{H}$ for the inner disk gas.

Our 1D model assumes the inner disk extends from the corotational radius at $0.015 \mathrm{AU}$ out to $0.024 \mathrm{AU}$. This width was determined from the radial distance travelled on a viscous timescale of $\sim 2.5$ years for the difference between epochs 2013 and 2010b, assuming a "typical" $\alpha=0.01$. We took a radially dependent power-law hydrogen density of power -2 . We created a composite radiation field to represent the joint contributions from the star and accretion shock. For the star we used ATLAS9 stellar models with a solar metallicity (Castelli \& Kurucz 2003) at the stellar effective temperature and luminosity of $T_{\text {eff }}=3850 \mathrm{~K}$ with $L_{*}=0.3 L_{\odot}$. We treated the accretion shock as a combination of ultraviolet (UV) and $\mathrm{X}$-ray emission. The UV emission was approximated by a stellar emission spectrum with a temperature and luminosity of $T_{\text {shock }}=8000 \mathrm{~K}$ with $L_{\text {shock }}=0.01 L_{\odot}$. We included a hard X-ray component, assuming a $10 \mathrm{MK}$ bremsstrahlung emission spectrum, with a luminosity of $L_{\mathrm{X}}=10^{30} \mathrm{erg} \mathrm{s}^{-1}$ between $0.3 \mathrm{keV}$ and $10 \mathrm{keV}$ (Rab et al. 2018). Accretion heating enabled us to use the Hextra keyword, as described in Sect. 11.9.4 of the Cloudy user manual, assuming $\alpha=0.01$, the stellar mass of $M_{*}=0.6 M_{\odot}$ (Sokal et al. 2018), and the radius set to $0.024 \mathrm{AU}$.

Cloudy includes a chemical network with atomic and molecular opacities that are appropriate for the hot, dense, dust-free region inside the inner dust rim, which enables a radiative transfer calculation in the absence of dust opacity. It is important to note that due to the high densities at this location, the chemistry does not behave as in a typical PDR, that is, the ionization of $\mathrm{H}$ is provided by charge exchange collisions, rather than photo- or collisional-ionization. Therefore, $\mathrm{C}^{0}$ and $\mathrm{H}^{0}$ coexist and they are the main $\mathrm{C}$ and $\mathrm{H}$ carriers despite the exposure to $\mathrm{UV}$ and X-rays or the expected self-shielding by $\mathrm{H}_{2}$. The inner disk chemistry is discussed extensively in the text of Sect. 3 and Figs. 3 and 4 of McClure (2019), but we also briefly summarize the salient mechanisms, the sources of systematic uncertainty in the final abundances, and a complete list of the model parameters in Appendix B.

A value of $\log \left(n_{\mathrm{H}}\right)=14.40 \mathrm{~cm}^{-3}$ at the inner edge of the disk model is required to fit the observed $\mathrm{Pa} \beta / \mathrm{Pa} \gamma=2.3$ flux ratio in the inner disk kinematic component from 2010b2010a. By 2013, the inner disk gas density increased to $\log \left(n_{\mathrm{H}}\right)=14.74 \mathrm{~cm}^{-3}$. This is consistent with the physical scenario derived from the hydrogen line profiles. We then fixed $\log \left(n_{\mathrm{H}}\right)$ to the 2013 value of $14.74 \mathrm{~cm}^{-3}$ and varied the atomic 
abundances of $\mathrm{C}, \mathrm{O}, \mathrm{S}$, and $\mathrm{Ca}$. Plots of the model fluxes with the elemental abundance for each element are shown in Fig. A.3. After confirming that the $\mathrm{Pa} \beta / \mathrm{P} \gamma$ ratio was still valid, we determined the $3 \sigma$ upper limits to the abundances of $\mathrm{N}, \mathrm{K}, \mathrm{Na}, \mathrm{Mg}$, $\mathrm{Fe}, \mathrm{Al}$, and $\mathrm{Si}$, which have undetected strong lines in this region. The resulting abundances are given in Table A.1.

\section{Results and discussion}

The results of this comprehensive elemental abundance measurement for the inner disk of a T Tauri star contradict the prediction of dust evolution models. All measured elements are depleted with respect to the Sun, with volatiles depleted by up to two orders of magnitude and refractories by up to five orders of magnitude. We note that our $\mathrm{C} / \mathrm{H}$ and $\mathrm{O} / \mathrm{H}$ values of $3.39 \times 10^{-6}$ and $1.60 \times 10^{-5}$, respectively, are consistent within our systematic uncertainties with the following $\mathrm{C} / \mathrm{H}$ and $\mathrm{O} / \mathrm{H}$ values for $\mathrm{TW}$ Hya that were recently found by Bosman \& Banzatti (2019): $3 \times 10^{-6}$ and $6 \times 10^{-6}$, respectively. Likewise, our N/H upper limit of $<1.6 \times 10^{-5}$ is depleted by a factor of $\sim 4$. This value encompasses the depleted N/H of $\sim 3 \times 10^{-6}$ inferred for TW Hya by van 't Hoff et al. (2017) from ALMA observations of $\mathrm{N}_{2} \mathrm{H}^{+}$. It is also consistent with an earlier finding of $\mathrm{N}$ depletion on the stellar surface of TW Hya, ranging between factors of 1.5 and 5 (Brickhouse et al. 2010). As is shown in Fig. 3, the accretion of primary atmospheres from this gas would not explain the enhanced giant planet atmospheric volatile abundances seen in our Solar System. However, the abundance pattern seen between the elements in TW Hya's gas is a close inverse of the CI chondrite abundance pattern, suggesting that the depletion could be caused by the retention of chondrite-like solids in the disk.

\subsection{Outer disk dust traps for $C, N$, and $S$}

Bosman \& Banzatti (2019) suggest that the C depletion in TW Hya's inner disk could result from either a dust trap at the $\mathrm{CO}$ snowline $(\sim 20 \mathrm{AU})$ or a $\mathrm{CO}_{2}$ trap close to the $\mathrm{H}_{2} \mathrm{O}$ snowline (4 AU). Due to the fact that our $\mathrm{C} / \mathrm{H}$ measurement is entirely within the dust sublimation rim, we can use previous measurements of $\mathrm{CO}$ and a simple analytic treatment (Appendix $\mathrm{C}$ ) to estimate the location where $\mathrm{C}$ is locked out.

First, we divided the disk into three zones (see Fig. 1), one exterior to the $\mathrm{CO}$ snowline (Zone 1), a second between the $\mathrm{CO}$ snowline and inner rim of the dust (Zone 2), and a third inside the inner rim of the dust (Zone 3). This division closely matches the dust traps found by Bosman \& Banzatti (2019), with the dust traps at the end of Zone 1 and in the middle of Zone 2. For each zone, we took a representative value of $\mathrm{C} / \mathrm{H}$. In Zone 3, a $\mathrm{C} / \mathrm{H}$ depletion factor of 79 was taken from the present work. For Zones 1 and 2, we calculated a $\mathrm{C} / \mathrm{H}$ ratio from the $\mathrm{C}^{18} \mathrm{O}$ measurements of Zhang et al. (2019). That work finds a minimum depletion factor for $\mathrm{CO} / \mathrm{H}_{2}$ of $\sim 78$ outside of the $\mathrm{CO}$ snowline, while the average depletion factor within the $\mathrm{CO}$ snowline is $\sim 20$, which is considered relative to the interstellar medium $\mathrm{CO} / \mathrm{H}_{2}$ value of $10^{-4}$. These values convert into $\mathrm{C} / \mathrm{H}$ values of $6.4 \times 10^{-7}$ and $2.5 \times 10^{-6}$, making the gross approximation that all gas phase $\mathrm{C}$ is in $\mathrm{CO}$.

Then, we consider that an infinitely thin parcel of gas and dust is transported from the outer edge of Zone 1 through each zone sequentially. The dust is composed of a CO ice component and a "higher temperature" component with carbon carriers that sublimate at higher temperatures than $\mathrm{CO}$, including $\mathrm{CO}_{2}$, organic residues, or graphite, for example. The dust is either locked into the disk in a given zone or allowed to pass through
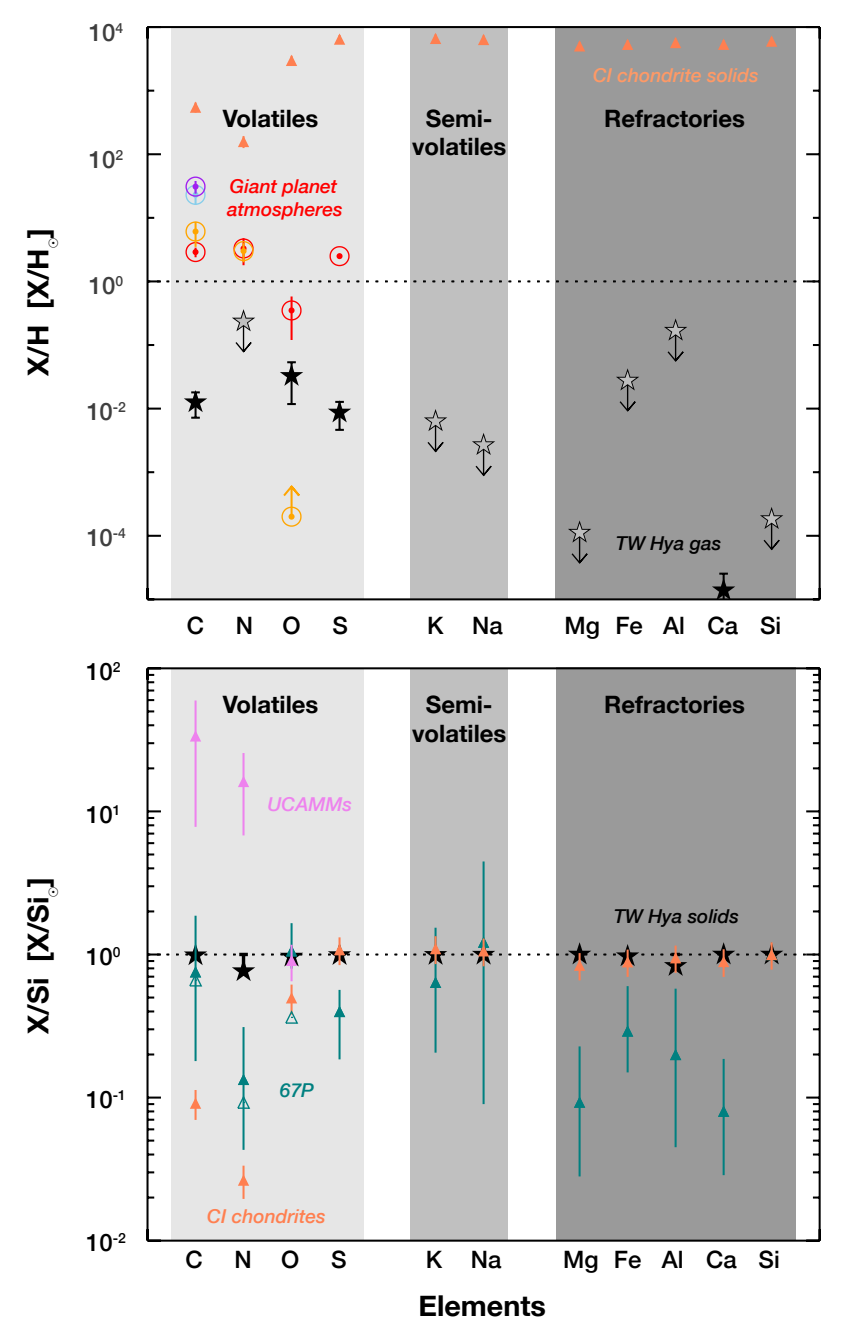

Fig. 3. Top: comparison of bulk elemental gas abundances inside TW Hya's dust sublimation rim (black stars, gray stars with arrows for upper limits), with atmospheric abundances of (Owen \& Encrenaz 2003) Jupiter (red circles), Saturn (orange circles), Uranus (light blue circle), and Neptune (purple circle), and CI chondrite solids (Lodders 2010, salmon triangles). All data are plotted with error bars. Abundances are with respect to hydrogen and are normalized to the solar abundances (Asplund et al. 2009), with the solar value indicated by the dotted horizontal line. Bottom: comparison of bulk elemental abundances of solids for: TW Hya (black stars), CI chondrites (Lodders 2010, salmon triangles), comet 67P (bulk, dust/ice 1, teal triangles; Bardyn et al. 2017; Rubin et al. 2019), and UCAMMs (violet triangles; Dartois et al. 2017; Mathurin et al. 2019).

into the next zone, where a portion of it sublimates. We assume a higher temperature component fraction of 0.83 , which is the refractory C fraction observed in comet 67P (Rubin et al. 2019), and we used Eqs. (C.5) and (C.10) to calculate the fraction of the initially available carbon dust that is locked into the disk that the parcel moves through in each zone. We find that $95.9 \%$ of the initial carbon dust mass is locked into the disk outside of the CO snowline (Zone 1). Therefore, the combined efficiency of any dust traps outside of that point is also $~ 96 \%$. Only $4.1 \%$ of the initial dust is transported into Zone 2 , with $0.7 \%$ of that dust in volatiles that sublimate. Between the $\mathrm{CO}$ snowline and the inner dust sublimation rim (Zone 2), an additional $3.06 \%$ of the initial dust mass is locked. Only $0.34 \%$ of the initial dust crosses the inner dust rim into Zone 3, where it sublimates. In total, only $1.04 \%$ of the initial Zone 1 carbon dust mass is returned 
to the gas phase that is to be accreted onto the central star. The agreement between our $\mathrm{C} / \mathrm{H}$ abundance in Zone 3 and that of Bosman \& Banzatti (2019), which is representative of the gap at $1 \mathrm{AU}$ in Zone 2 and between the $\mathrm{H}_{2} \mathrm{O}$ and $\mathrm{CO}_{2}$ ice snowlines and the silicate dust trap, suggests that $3 \%$ of the initial dust locked in Zone 2 is located beyond the $1 \mathrm{AU}$ gap. The efficiency of locking in traps between 1.5 and $20 \mathrm{AU}$ is then $90 \%$.

Most of the uncertainty in the above estimate depends on the higher temperature carbon fraction. Reasonable values range from 0.5 , which would lock $99 \%$ of the initial carbon mass within Zone 1, at the expense of Zone 2. By decreasing the $\mathrm{CO} / \mathrm{H}_{2}$ depletion factor from 20 to 15 , the value observed by Zhang et al. (2019) at 4 AU would reduce the locked dust mass beyond the $\mathrm{CO}$ snowline to $94 \%$ of the initial dust mass, suggesting that even if $\mathrm{CO}$ is converted into $\mathrm{CO}_{2}$ ice, most of that material is already efficiently trapped in the outer disk rings. Locking solids chemically isolates the inner disk, thus preventing the replenishment of higher temperature $\mathrm{C}$ and potentially leading to Earth-like C abundances (Klarmann et al. 2018; Anderson et al. 2017) for forming terrestrial planets.

A quantative analysis of the $\mathrm{N}$ depletion validates our $\mathrm{C}$ transport model and choice of $f_{\mathrm{r}}$. The $\mathrm{N} / \mathrm{H}$ found by van 't Hoff et al. (2017) is only $4 \%$ of the solar abundance, that is, the same fraction of $\mathrm{N}$ is missing as we find for $\mathrm{C}$ beyond the CO snowline in our model using $f_{\mathrm{r}}=0.83$. Since $\mathrm{N}_{2}$ is likely the dominant solid $\mathrm{N}$-carrier in the outer disk and as its midplane snowline at $\sim 18 \mathrm{~K}$ (Piso et al. 2016) should be just outside of the $\mathrm{CO}$ snowline, then the $\mathrm{N}_{2}$ must also be trapped in the three submillimeter rings beyond $30 \mathrm{AU}$.

In contrast with TW Hya, several other disks show no evidence of $\mathrm{C}$ or $\mathrm{N}$ gas depletion. HD 163296 has an enhanced $\mathrm{C} / \mathrm{H}$ in the gas interior to the $\mathrm{CO}$ snowline (Zhang et al. 2020) due to radial drift, while IM Lup shows depletions of a factor of 4 at most (Cleeves et al. 2018). For IM Lup, the gas temperature structure puts the $\mathrm{N}_{2}$ snowline at $>250$ AU (Cleeves et al. 2016, Fig. 9), which is well outside of the outermost dust ring in IM Lup at $134 \mathrm{AU}$ (Huang et al. 2018). Therefore, only a little bit of $\mathrm{N}_{2}$ ice is trapped in this disk and gaseous $\mathrm{N}$ is not as depleted. A similar lack of dust traps beyond the $\mathrm{N}_{2}$ snowline could account for the ISM N abundance found by Anderson et al. (2019) for a set of 5-11 Myr disks in upper Sco; however, these disks lack the same high spatial resolution continuum observations that enabled the ring detections in IM Lup and TW Hya. For HD 163296, a ring with a deep gap does sit at $100 \mathrm{AU}$, just outside the CO snowline at $70 \mathrm{AU}$, which apparently does not trap dust efficiently (Isella et al. 2018). However, while TW Hya's outer disk gaps are not as deep as in HD 163296, it does have three of them, which may explain their large cumulative trapping efficiency. Alternatively, if the dust in the rings of TW Hya has grown larger than in HD 163296, it may be filtered out more efficiently (Rice et al. 2006) or planetesimals may have formed, meaning that they would no longer drift efficiently regardless of trapping.

As a further check as to the locking location, in the bottom panel of Fig. 3, we compare the bulk atomic composition of the solids left behind in TW Hya's disk with the abundances of some primitive Solar System bodies, which roughly originate in each of the three zones, for example, CI chondrites (Lodders 2010, Zone 3), comet 67P (Bardyn et al. 2017; Rubin et al. 2019, Zone 2), and ultra-carbonaceous Antarctic micro-meteorites (Dartois et al. 2017; Mathurin et al. 2019, UCAMMs, Zone 1). The TW Hya solids are all nearly solar; and within the uncertainties, chondritic abundances are generally a good match for the refractory and semi-volatile elements as well as S. However, chondrites underproduce TW Hya's $\mathrm{C}, \mathrm{N}$, and $\mathrm{O}$ abundances by an order of magnitude in the case of the former two. Comet 67P and the UCAMMs are more abundant in all three of these elements, but with opposite abundance patterns, that is, there is low N/O in comets and high N/O in UCAMMs. A crude check, fitting a blend of abundances from CI chondrites and either Comet 67P or UCAMMs, or a combination of the two, to TW Hya's solid abundances, confirms that neither 67P nor UCAMMs on their own provide a good fit to $\mathrm{C}, \mathrm{N}$, or $\mathrm{O}$. The former overproduces $\mathrm{O}$ and underproduces $\mathrm{N}$, while the latter underproduces $\mathrm{N}$ and $\mathrm{O}$, and both fits are poor overall $\left(\chi^{2} \sim 5000-8000\right)$. Interestingly, a combination of all three bodies succeeds in fitting $\mathrm{C}$ and $\mathrm{O}$, with a $\chi^{2} \sim 3$, but not $\mathrm{N}$.

There are several potential explanations as to the apparent "excess" of nitrogen in TW Hya's solids. The UCAMMs are thought to originate from differentiated parent bodies beyond the $\mathrm{N}_{2}$ snowline in which $\mathrm{N}_{2}$ was converted to higher temperature residues (Dartois et al. 2017), but they are ultimately recovered in Antarctica. These residues are more stable against sublimation than the original $\mathrm{N}_{2}$ ice, but is is possible that they encountered some loss on atmospheric entry, artificially lowering the observed abundances. Alternatively, there may be differences between the dust trapping patterns in the Solar System and TW Hya that produce different types of primitive bodies; that is to say, CI chondrites, comet 67P, and UCAMMs may not be an appropriate basis set for TW Hya's solids. This is generally true to some extent, particularly as we have only used one comet, 67P, to represent the entire cometary class due to a lack of comprehensive elemental abundances for additional comets. Finally, the primitive bodies represent the cumulative elements locked into solids at a particular disk radius over the lifetime of the solar nebula, while TW Hya's solid abundances represent a snapshot of the material that has been locked out of a moving parcel of gas over multiple radii and a later range of times, from 2-8 Myr based on viscous accretion timescales. If the N-rich dust trap formed later in TW Hya's lifetime than it did in the solar nebula, then the net effect on the cumulative $\mathrm{N}$ abundances in the primitive bodies would be diluted.

Unlike N, S can be found in both volatile molecules, for example, $\mathrm{H}_{2} \mathrm{~S}$, or minerals that contain more refractory elements, that is, FeS. An analysis of the atmospheres of accreting Herbig $\mathrm{AeBe}$ stars with disks suggests that of the total S content, $<1 \%$ is in gaseous form, with $89 \pm 8 \%$ of $\mathrm{S}$ is in refractory minerals (Kama et al. 2019) and the remainder in ices. The depletion of S here is consistent with an $89 \%$ refractory fraction; if the trap at $2 \mathrm{AU}$ lets through $0.3 \%$ of the total initial dust mass, as for the $\mathrm{C}$ transport model, then the sublimation of volatile ice with an initial fraction of $11 \%$ in that dust can account for all of the gas phase $\mathrm{S}$ that we see in the inner disk. The mineral sulphides pass through the $1 \mathrm{AU}$ gap into the inner, refractory disk, which is discussed below.

\subsection{Inner disk refractory dust trap}

One of the refractory elements, $\mathrm{Ca}$, was firmly detected in the gas phase with a depletion of nearly five orders of magnitude, relative to the solar value. While more depletion of refractories than volatiles is to be expected due to rocky cores in all icy dust grains, five orders of magnitude seems extreme at first glance. However, two of the upper limits to the abundances of the other refractory elements, $\mathrm{Mg}$ and $\mathrm{Si}$, are depleted by approximately $10^{-4}$, which roughly supports these types of low values. The latter is qualitatively consistent with previous findings of weaker $\mathrm{Si}$ relative to C or N in TW Hya's accretion streams (Ardila et al. 2013). Our 
extreme depletions suggest that there is an additional dust trap interior to the sublimation point of most species considered for our higher temperature carbon component, where the bare silicate grains are prevented from sublimating.

An obvious choice for this trap is the dust ring inside of 0.5 AU, detected with ALMA (Andrews et al. 2016). The inner disk temperature and density structures calculated selfconsistently in hydrostatic equilibrium normally reach the silicate sublimation temperature of $1400 \mathrm{~K}$ at $\sim 0.1 \mathrm{AU}$ in M0 T Tauri stars with mass accretion rates $>10^{-9} M_{\odot} \mathrm{yr}^{-1}$ (e.g., GO Tau in McClure et al. 2013b, Fig. 10). However, using nearand mid-infrared (IR) interferometry, Menu et al. (2014) find the inner rim for TW Hya at $0.3 \mathrm{AU}$ instead, suggesting that the inner edge is associated with a pressure maximum rather than the silicate sublimation front. Blocking accretion or drift of large silicate grains at $0.3 \mathrm{AU}$ would retain the elements $\mathrm{Mg}, \mathrm{Si}$, and $\mathrm{Ca}$ in the disk. The cumulative amount of the initial dust mass passing through the three submillimeter rings outside of the $\mathrm{CO}$ and $\mathrm{N}_{2}$ snowlines is $\sim 4 \%$, and the trap near the water snowline only allows $10 \%$ of that first $4 \%$ of the dust to continue through. To reproduce the fraction of initial $\mathrm{Si}, \mathrm{Mg}$, and $\mathrm{Ca}$ that returned to the inner disk gas of $\sim 10^{-4}-2 \times 10^{-5}$, only $4.6-0.6 \%$ of the dust in the inner $0.3-0.5 \mathrm{AU}$ can pass through the $0.3 \mathrm{AU}$ dust trap and sublimate. This suggests that the trapping efficiency is inversely proportion to the disk radius, with a $96 \%$ trapping efficiency cumulatively over the outer three submillimeter rings, $90 \%$ at the $2 \mathrm{AU}$ ring, and $\sim 95-99 \%$ at the $0.3 \mathrm{AU}$ ring.

If the refractory $\mathrm{C}$ within the $0.3-0.5 \mathrm{AU}$ ring is efficiently removed by photolysis or oxidation, as is suggested by Anderson et al. (2017), then the high trapping efficiency of the $2 \mathrm{AU}$ and outer submillimeter dust traps cuts off $\mathrm{C}$ replenishment by drifting solids, as is proposed by Klarmann et al. (2018). Should the innermost, refractory trap of TW Hya eventually form terrestrial planets, they would form from very dry and poor in $\mathrm{C}$ and $\mathrm{N}$ dust, possibly resulting in Earth-like depletions of these elements.

\section{Conclusions}

To summarize, we piloted a novel method to determine the bulk atomic composition of unseen solids locked at the midplanes of protoplanetary disks, using the TW Hya disk. The volatile elements in this system are depleted by a factor of $\sim 100$ with respect to the solar value, while the refractories are depleted by up to five orders of magnitude. The high degree of depletion and the difference between the depletion factors of the volatile and refractory elements can be explained if all of the submillimeter rings host dust traps, including the truncated ring from 0.3-0.5 AU. A simple model for the efficiency of dust retention in these traps finds that the inner two dust traps at 0.3 and 2 AU have a high efficiency ( $\geq 90 \%$ each), while the three submillimeter rings beyond the midplane $\mathrm{CO}$ and $\mathrm{N}_{2}$ snowlines are collectively $\sim 96 \%$ and less individually. Such a high retention efficiency, relative to other disks, may be due to the positioning of the rings relative to individual molecular snowlines as well as the age and maximum grain size in the system. In any event, only a small fraction of the elements in dust end up accreted onto the central star. These results, combined with theoretical modeling of refractory $\mathrm{C}$ destruction, suggest that any terrestrial planets forming in the trap within TW Hya's inner disk from the remaining solids will be relatively "dry" and C-poor, similar to those in our Solar System.

Acknowledgements. This publication is part of a project that has received funding from the European Union's Horizon 2020 research and innovation pro- gram under the Marie Skłodowska-Curie grant agreement ICED No. 749864 (M.M.). M.K. was supported by the University of Tartu ASTRA project 2014 2020.4.01.16-0029 KOMEET, financed by the EU European Regional Development Fund. We thank the anonymous referee for comments that significantly improved the manuscript. C. D. acknowledges support by Nederlandse Organisatie voor Wetenschappelijk Onderzoek (NWO), project no. ALW-GO/15-01.

\section{References}

Anderson, D. E., Bergin, E. A., Blake, G. A., et al. 2017, ApJ, 845, 13 Anderson, D. E., Blake, G. A., Bergin, E. A., et al. 2019, ApJ, 881, 127 Andrews, S. M., Wilner, D. J., Zhu, Z., et al. 2016, ApJ, 820, L40 Ardila, D. R., Herczeg, G. J., Gregory, S. G., et al. 2013, ApJS, 207, 1 Asplund, M., Grevesse, N., Sauval, A. J., \& Scott, P. 2009, ARA\&A, 47, 481 Banzatti, A., \& Pontoppidan, K. M. 2015, ApJ, 809, 167

Bardyn, A., Baklouti, D., Cottin, H., et al. 2017, MNRAS, 469, S712 Bergin, E. A., Cleeves, L. I., Gorti, U., et al. 2013, Nature, 493, 644 Bergin, E. A., Blake, G. A., Ciesla, F., Hirschmann, M. M., \& Li, J. 2015, Proc. Natl. Acad. Sci., 112, 8965

Bosman, A. D., \& Banzatti, A. 2019, A\&A, 632, L10

Brickhouse, N. S., Cranmer, S. R., Dupree, A. K., Luna, G. J. M., \& Wolk, S. 2010, ApJ, 710, 1835

Castelli, F., \& Kurucz, R. L. 2003, in Modelling of Stellar Atmospheres, eds. N. Piskunov, W. W. Weiss, \& D. F. Gray, IAU Symp., 210, A20

Cleeves, L. I., Öberg, K. I., Wilner, D. J., et al. 2016, ApJ, 832, 110 Cleeves, L. I., Öberg, K. I., Wilner, D. J., et al. 2018, ApJ, 865, 155 Dartois, E., Chabot, M., Pino, T., et al. 2017, A\&A, 599, A130 Drake, J. J., Testa, P., \& Hartmann, L. 2005, ApJ, 627, L149 Du, F., Bergin, E. A., \& Hogerheijde, M. R. 2015, ApJ, 807, L32 Dupree, A. K., Brickhouse, N. S., Cranmer, S. R., et al. 2012, ApJ, 750, 73 Ferland, G. J., Chatzikos, M., Guzmán, F., et al. 2017, Rev. Mex. Astron. Astrofis., 53, 385

France, K., Schindhelm, E., Herczeg, G. J., et al. 2012, ApJ, 756, 171

Hartmann, L., Herczeg, G., \& Calvet, N. 2016, ARA\&A, 54, 135

Heays, A. N., Visser, R., Gredel, R., et al. 2014, A\&A, 562, A61

Herczeg, G. J., Linsky, J. L., Valenti, J. A., Johns-Krull, C. M., \& Wood, B. E. 2002, ApJ, 572, 310

Hily-Blant, P., Magalhaes de Souza, V., Kastner, J., \& Forveille, T. 2019, A\&A, 632, L12

Hogerheijde, M. R., Bergin, E. A., Brinch, C., et al. 2011, Science, 334, 338 Huang, J., Andrews, S. M., Cleeves, L. I., et al. 2018, ApJ, 852, 122 Isella, A., Huang, J., Andrews, S. M., et al. 2018, ApJ, 869, L49 Jermyn, A. S., \& Kama, M. 2018, MNRAS, 476, 4418 Kama, M., Folsom, C. P., \& Pinilla, P. 2015, A\&A, 582, L10 Kama, M., Shorttle, O., Jermyn, A. S., et al. 2019, ApJ, 885, 114 Kamp, I., Thi, W. F., Meeus, G., et al. 2013, A\&A, 559, A24 Kingdon, J. B., \& Ferland, G. J. 1996, ApJS, 106, 205 Kingdon, J. B., \& Ferland, G. J. 1999, ApJ, 516, L107 Klarmann, L., Ormel, C. W., \& Dominik, C. 2018, A\&A, 618, L1 Krijt, S., Schwarz, K. R., Bergin, E. A., \& Ciesla, F. J. 2018, ApJ, 864, 78 Lodders, K. 2010, Astrophys. Space Sci. Proc., 16, 379 Madhusudhan, N. 2012, ApJ, 758, 36

Mathurin, J., Dartois, E., Pino, T., et al. 2019, A\&A, 622, A160 McClure, M. K. 2019, A\&A, 632, A32

McClure, M. K., Calvet, N., Espaillat, C., et al. 2013a, ApJ, 769, 73 McClure, M. K., D’Alessio, P., Calvet, N., et al. 2013b, ApJ, 775, 114 Menu, J., van Boekel, R., Henning, T., et al. 2014, A\&A, 564, A93 Owen, T., \& Encrenaz, T. 2003, Space Sci. Rev., 106, 121 Piso, A.-M. A., Pegues, J., \& Öberg, K. I. 2016, ApJ, 833, 203 Rab, C., Güdel, M., Woitke, P., et al. 2018, A\&A, 609, A91

Rice, W. K. M., Armitage, P. J., Wood, K., \& Lodato, G. 2006, MNRAS, 373 , 1619

Rubin, M., Altwegg, K., Balsiger, H., et al. 2019, MNRAS, 489, 594 Schwarz, K. R., Bergin, E. A., Cleeves, L. I., et al. 2016, ApJ, 823, 91 Shaw, G., Ferland, G. J., Abel, N. P., Stancil, P. C., \& van Hoof, P. A. M. 2005, ApJ, 624, 794

Simcoe, R. A., Burgasser, A. J., Schechter, P. L., et al. 2013, PASP, 125, 270

Sokal, K. R., Deen, C. P., Mace, G. N., et al. 2018, ApJ, 853, 120 van Boekel, R., Henning, T., Menu, J., et al. 2017, ApJ, 837, 132 van 't Hoff, M. L. R., Walsh, C., Kama, M., Facchini, S., \& van Dishoeck, E. F. 2017, A\&A, 599, A101

Vernet, J., Dekker, H., D’Odorico, S., et al. 2011, A\&A, 536, A105

Zhang, K., Pontoppidan, K. M., Salyk, C., \& Blake, G. A. 2013, ApJ, 766, 82 Zhang, K., Bergin, E. A., Blake, G. A., Cleeves, L. I., \& Schwarz, K. R. 2017, Nat. Astron., 1, 0130

Zhang, K., Bergin, E. A., Schwarz, K., Krijt, S., \& Ciesla, F. 2019, ApJ, 883, 98 Zhang, K., Bosman, A. D., \& Bergin, E. A. 2020, ApJ, 891, L16 


\section{Appendix A: Observation and data reduction details}

One spectrum of TW Hya was obtained with the FIRE spectrograph (Simcoe et al. 2013) at Las Campanas $(R \sim 6000$, $0.8<\lambda<2.5 \mu \mathrm{m})(30)$ on January 2, 2013 (PI McClure). Two $6.0 \mathrm{~s}$ exposures in the Fowler 1 read mode were obtained with the $0.6^{\prime \prime}$ slit under $0.45^{\prime \prime}$ seeing at an airmass of 1.009 in excellent conditions. We obtained the usual suite of arc lamp and flat-field calibrations and extracted the FIRE spectrum using the standard FIREhose pipeline, with MCN 7202 as the telluric calibrator star. The other two spectra were obtained with VLT
X-shooter (Vernet et al. 2011) (visible: $0.8-1 \mu \mathrm{m}, R \sim 18000$; near-IR arm: $1-2.5 \mu \mathrm{m}, R \sim 11000)$ on April 7, 2010 and May 3, 2010. We corrected ESO's Phase 3 reduced data for telluric absorption using the MolecFit software package. Spectra from all three epochs were corrected for their heliocentric velocities, and the X-shooter spectra were convolved down to the resolution of the FIRE spectrum. The processed spectra, before and after local continuum subtraction (as described in Sect. 2), are shown below. A comparable spectrum of hydrogen is shown in the main text (Fig. 2). The integrated line fluxes are given in Table A.1.
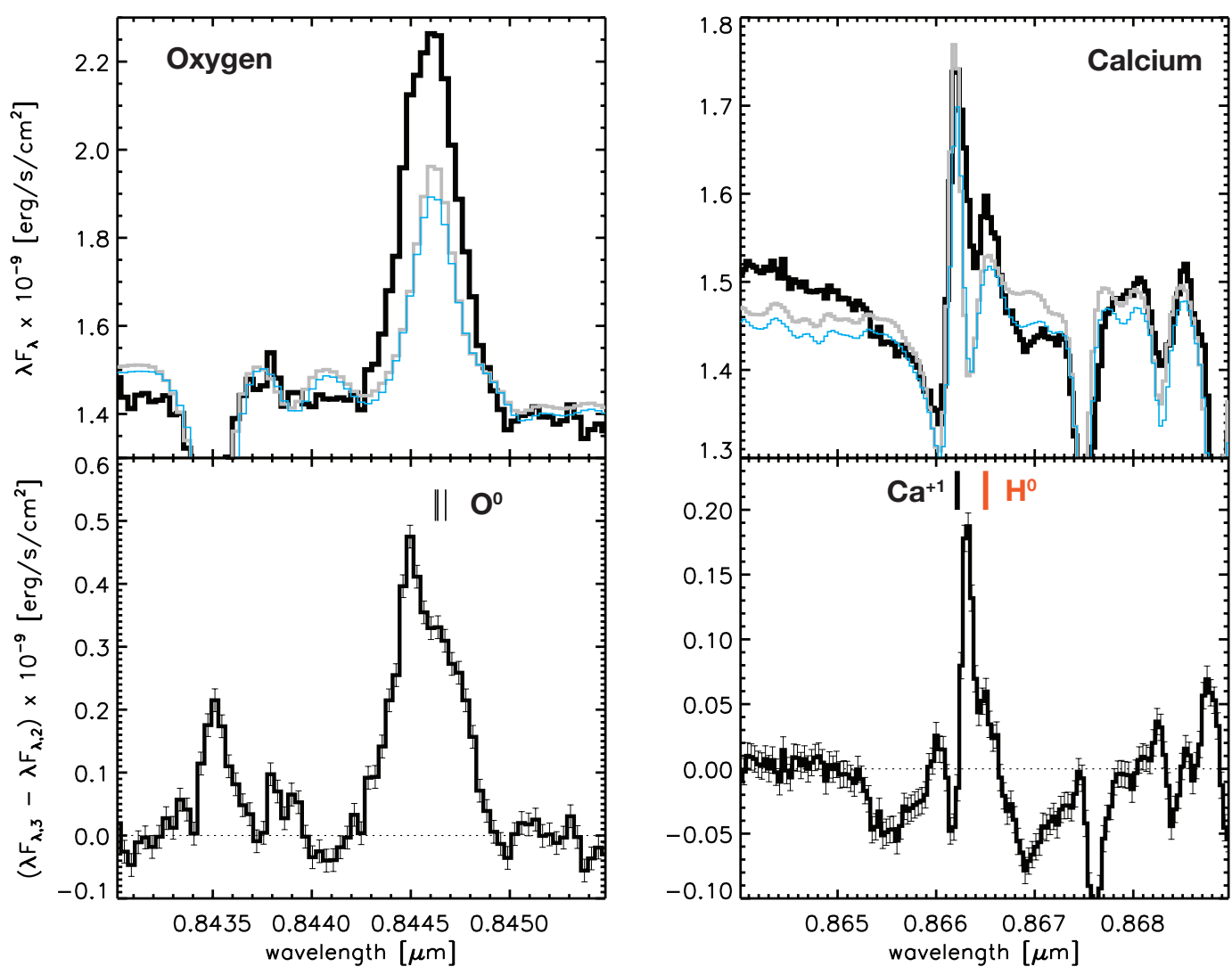

Fig. A.1. Top: comparison of the three observed epochs of TW Hya: 2010a (cyan, thin line), 2010b (gray, medium line), and 2013 (black, thick line). The oxygen line (left) is a triple line blend, and the calcium line (right) is blended with an $\mathrm{H}^{0}$ Paschen series line and an absorption artifact from the telluric correction of that line, which we fit out before determining the integrated flux. Bottom: residual spectra after subtraction of epoch 2 from epoch 3 . 
Table A.1. Observed line fluxes and modeled abundances.

\begin{tabular}{|c|c|c|c|c|c|}
\hline Atomic species & $\begin{array}{l}\text { Line } \\
{[\mu \mathrm{m}]}\end{array}$ & $\begin{array}{c}F_{\text {int }} \\
{\left[\operatorname{erg~s}^{-1} \mathrm{~cm}^{2}\right]}\end{array}$ & $S / N$ & $F_{\mathrm{X}} / F_{\mathrm{Pa} \gamma}$ & $\mathrm{X} / \mathrm{H}\left[(\mathrm{X} / \mathrm{H})_{\odot}\right]$ \\
\hline $\mathrm{H}^{0}(\mathrm{~Pa} \gamma)$ & 1.09381 & $7.0 \times 10^{-9}$ & 187 & - & \\
\hline $\mathrm{H}^{0}(\mathrm{~Pa} \beta)$ & 1.28181 & $2.0 \times 10^{-8}$ & 1090.5 & $2.857 \pm 0.016$ & $\log \left(n_{\mathrm{H}}\right)=14.74 \pm 0.02$ \\
\hline $\mathrm{C}^{0}$ & 1.06831 & $3.8 \times 10^{-10}$ & 18.3 & $0.0543 \pm 0.0030$ & $1.26 \pm 0.54 \times 10^{-2}$ \\
\hline $\mathrm{N}^{0}$ (blend) & 1.04072 & $<2.5 \times 10^{-10}$ & $<3$ & $<0.0073$ & $<2.35 \times 10^{-1}$ \\
\hline $\mathrm{O}^{0}$ (blend) & 0.84464 & $1.7 \times 10^{-9}$ & 58.7 & $0.2429 \pm 0.0043$ & $3.27 \pm 2.09 \times 10^{-2}$ \\
\hline $\mathrm{Na}^{0}$ & 1.70311 & $<4.7 \times 10^{-11}$ & $<3$ & $<0.0067$ & $<2.67 \times 10^{-3}$ \\
\hline $\mathrm{Mg}^{0}$ & 1.71087 & $<4.7 \times 10^{-11}$ & $<3$ & $<0.0067$ & $<1.12 \times 10^{-4}$ \\
\hline $\mathrm{Al}^{0}$ & 1.67189 & $<1.1 \times 10^{-10}$ & $<3$ & $<0.0157$ & $<1.68 \times 10^{-1}$ \\
\hline $\mathrm{Si}^{0}$ & 1.58884 & $<9.8 \times 10^{-11}$ & $<3$ & $<0.0140$ & $<1.83 \times 10^{-4}$ \\
\hline $\mathrm{S}^{0}$ (blend) & 1.04568 & $5.6 \times 10^{-10}$ & 39.8 & $0.0800 \pm 0.0021$ & $8.70 \pm 4.06 \times 10^{-3}$ \\
\hline $\mathrm{K}^{0}$ & 1.51631 & $<4.7 \times 10^{-11}$ & $<3$ & $<0.0067$ & $<6.33 \times 10^{-3}$ \\
\hline $\mathrm{Ca}^{+1}$ & 0.86621 & $8.7 \times 10^{-10}$ & 93.1 & $0.1240 \pm 0.0015$ & $1.39 \pm 1.15 \times 10^{-5}$ \\
\hline $\mathrm{Fe}^{+1}$ & 1.64355 & $<4.0 \times 10^{-11}$ & $<3$ & $<0.0057$ & $<2.72 \times 10^{-2}$ \\
\hline
\end{tabular}

Notes. The uncertainties on the modeled abundances are dominated by a systematic $20 \%$ uncertainty on the synthetic line fluxes, which results from a factor of $\sim 2-4$ uncertainty on the charge exchange rate coefficients, as is discussed in Sect. 3.3.4 of Hazy, the Cloudy users' manual. Abundances are with respect to the Asplund et al. (2009) solar values.
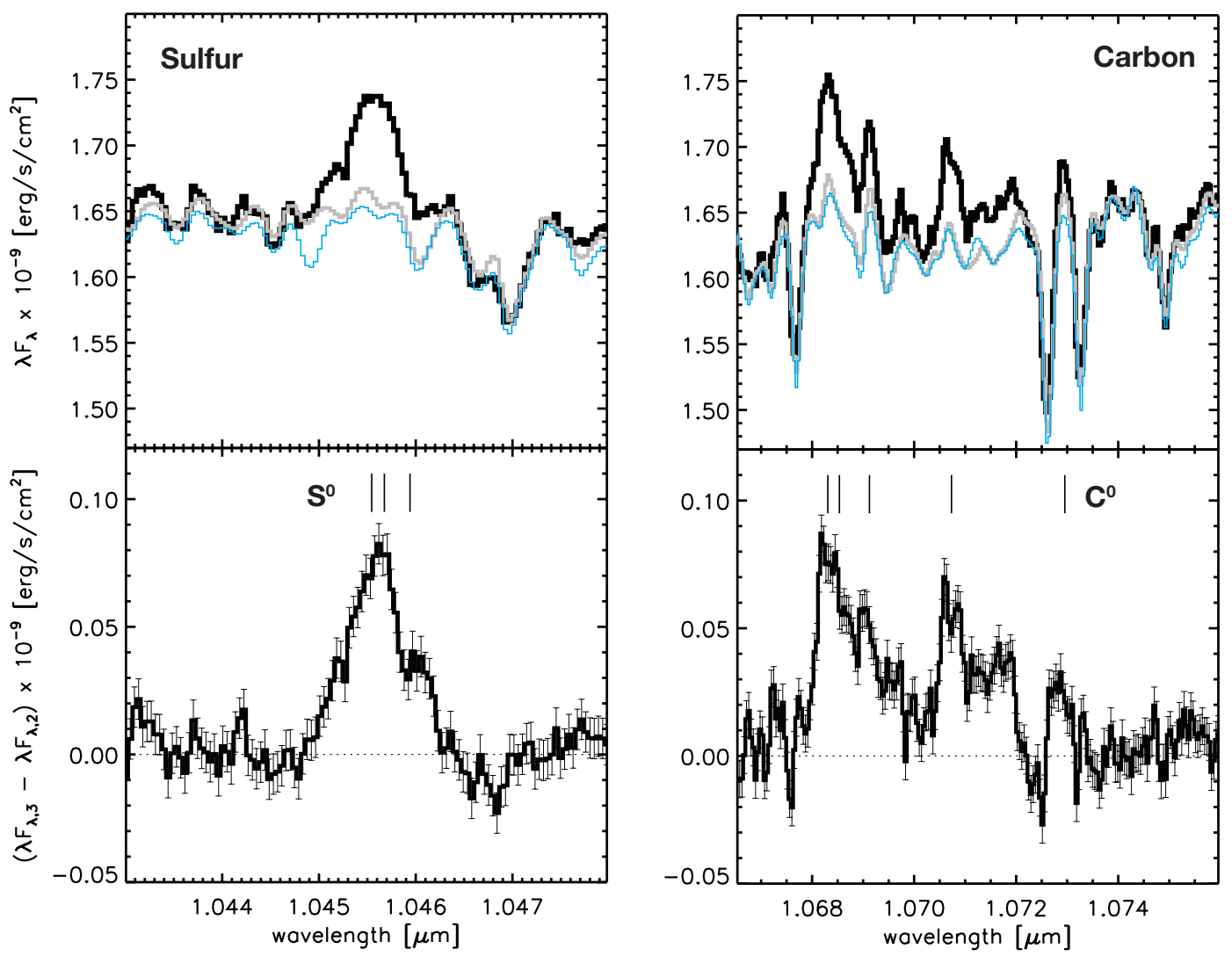

Fig. A.2. Same as Fig. A.1, but for S (left $)$ and C (right). The integrated flux of the S line is includes the three-line blend, while the C flux includes only the first three lines. The line styles are the same as in Fig. A.1. 
M. K. McClure et al.: Measuring the atomic composition of planetary building blocks
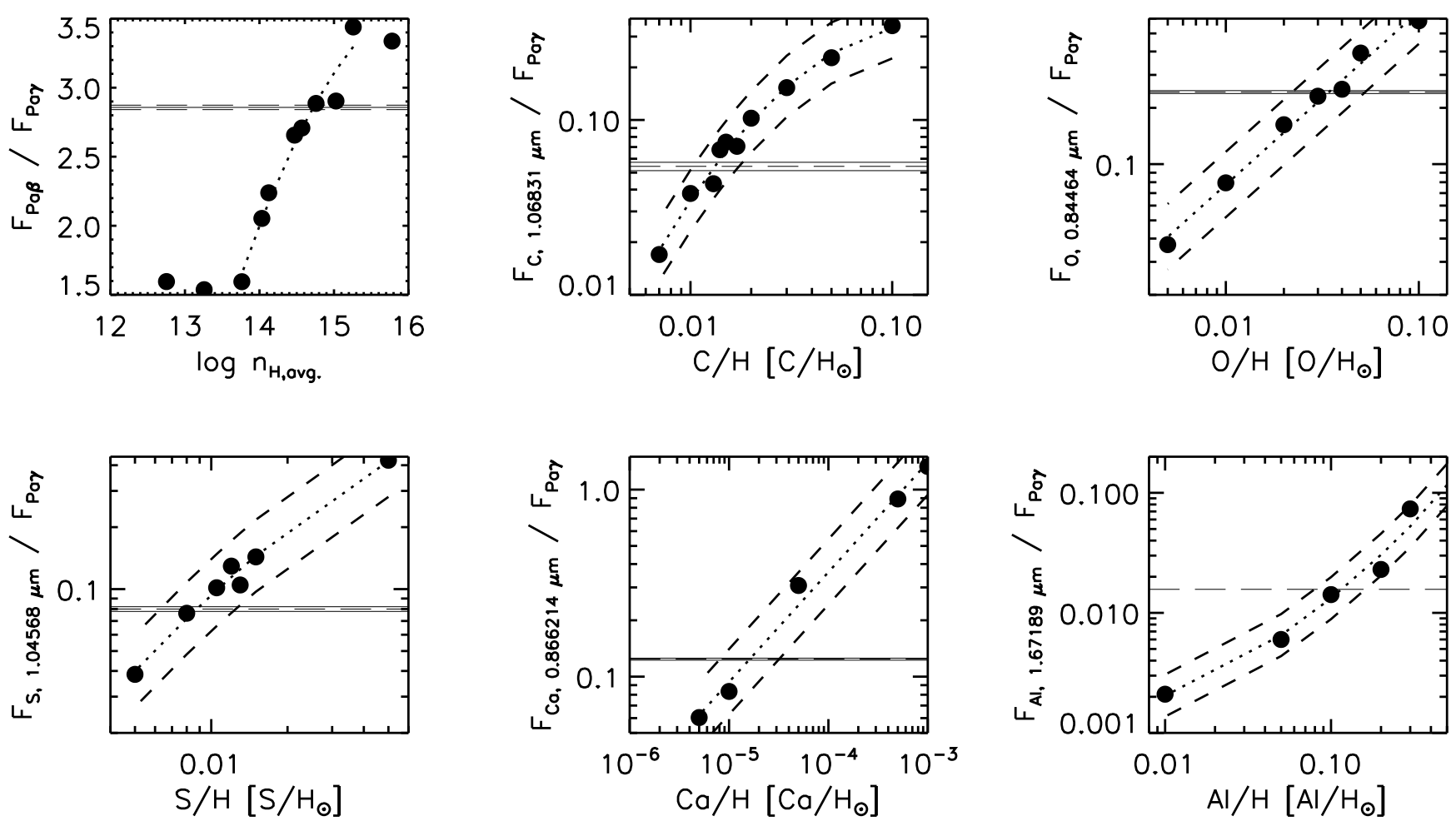

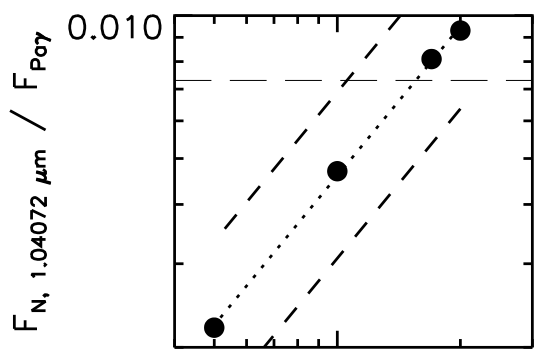

0.1

$\mathrm{N} / \mathrm{H}\left[\mathrm{N} / \mathrm{H}_{\odot}\right]$

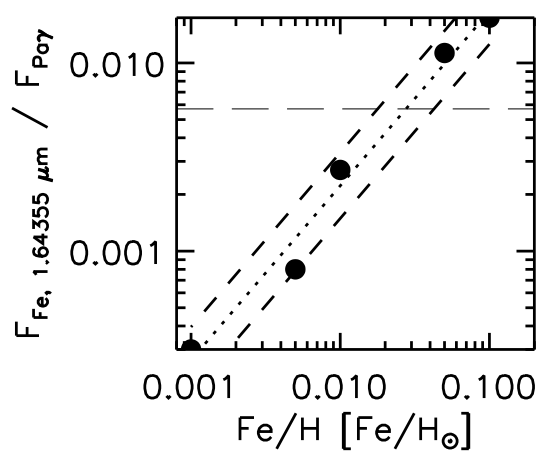

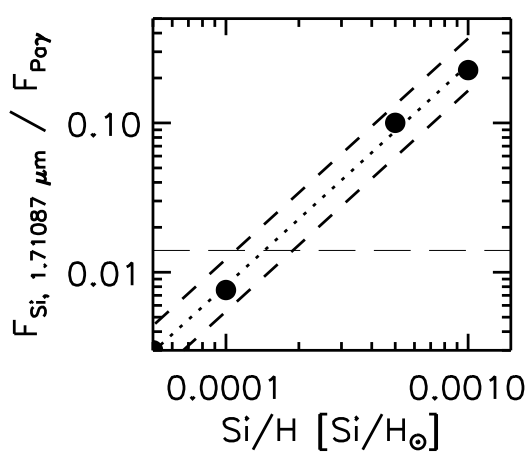
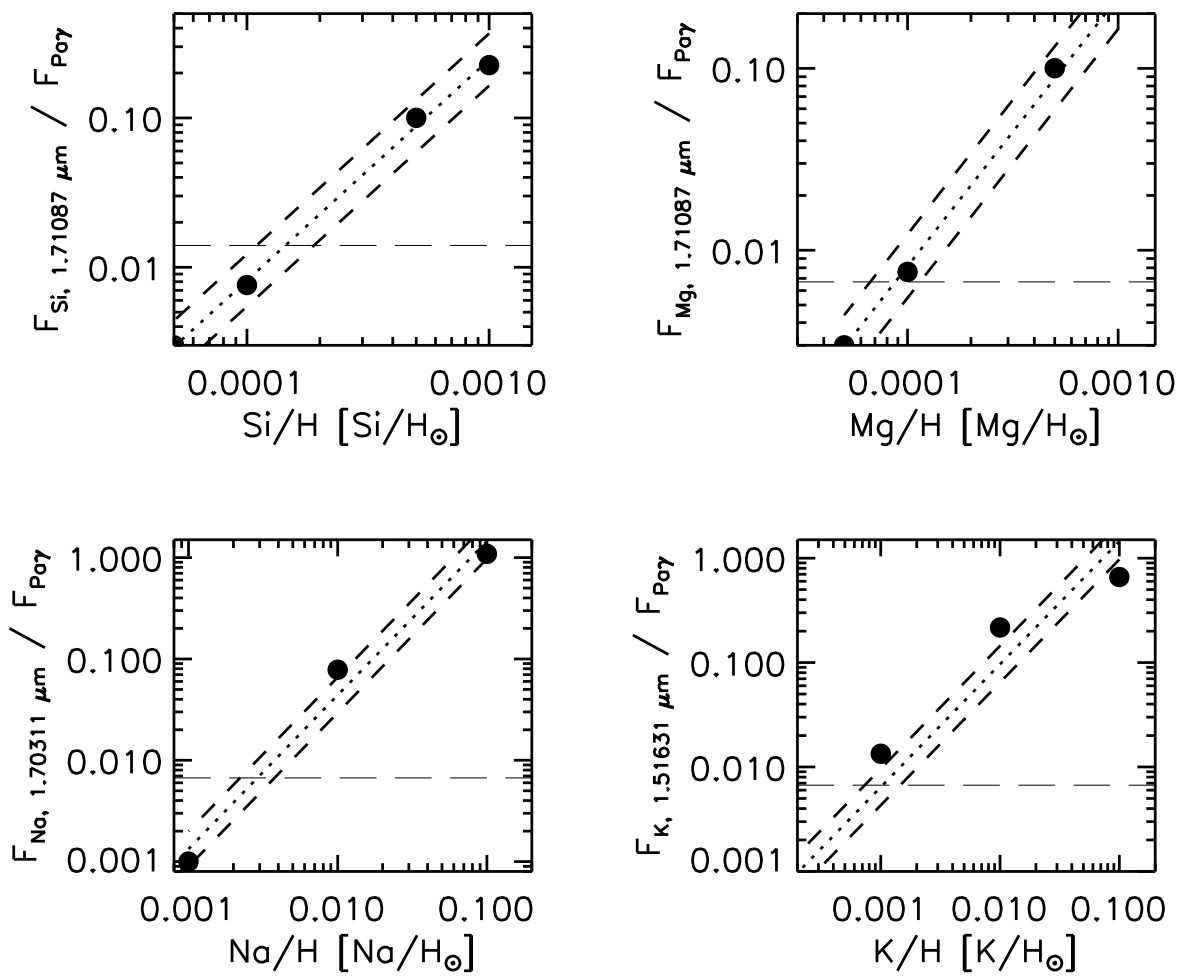

Fig. A.3. Upper left corner: model flux ratios between $\mathrm{Pa} \beta$ and $\mathrm{Pa} \gamma$ ( $y$-axis) versus $n_{\mathrm{H}}$ ( $x$-axis), compared with the observed flux ratio and error bars (horizontal lines). All other panels: model flux ratios of studied atomic lines relative to Pay ( $y$-axis) versus elemental abundance relative to $\mathrm{H}$ in solar units ( $x$-axis) for all elements in this study. The diagonal dashed lines indicate the systematic error in the models due to the charge exchange rate coefficients, as is discussed in Appendix B. Observational flux ratios are indicated (horizontal lines) with error bars for the four elements with $>3 \sigma$ detections $(\mathrm{C}, \mathrm{O}, \mathrm{S}$, and $\mathrm{Ca}$ ), while the $3 \sigma$ upper limits are indicated for the other elements. 


\section{Appendix B: Inner disk chemical model mechanisms and uncertainties}

Our 1D inner disk model uses version 17.02 of Cloudy (Ferland et al. 2017). As discussed in Sect. 3.3 of McClure (2019), the high midplane gas densities at $0.024 \mathrm{AU}\left(n_{\mathrm{H}} \sim\right.$ $\left.10^{15}\left[\mathrm{~cm}^{-3}\right], N_{\mathrm{H}} \sim 10^{26}\left[\mathrm{~cm}^{-2}\right]\right)$ lead to a chain of reactions that ultimately produce a mostly neutral gas population, but with a large fraction of free electrons donated mainly by hydrogen itself.

Since the physics and chemistry of molecular hydrogen is critical to understanding the atomic $\mathrm{H}$ emission, we enabled the larger $\mathrm{H}_{2}$ molecule module in Cloudy (Shaw et al. 2005). The collisional dissociation of $\mathrm{H}_{2}$ is efficient at these high densities; however, the formation of $\mathrm{H}_{2}$ is hampered due to the absence of dust inside the silicate sublimation rim (at $0.3 \mathrm{AU}$ ). Therefore, a large population of $\mathrm{H}^{0}$ exists. In turn, at these densities, $\mathrm{H}^{+1}$ is efficiently created through charge exchange reactions, particularly with sulfur as the collision partner. The fraction of hydrogen in $\mathrm{H}^{+1}$ is $\sim 10^{-3.5}$ and, therefore, $\mathrm{H}$ is the major contributor to the electron density, unlike a PDR where $\mathrm{C}^{+1}$ dominates. The recombination to $\mathrm{H}^{0}$ produces Lyman photons. However, at these large column densities, the gas is optically thick to UV radiation, so the Lyman photons are absorbed locally by the remaining population of $\mathrm{H}_{2}$, causing it to enter an excited electronic state. Collisional de-excitation of the $\mathrm{H}_{2}$ then heats the surrounding gas to $\sim 6000 \mathrm{~K}$. The net result is that at this location in the disk midplane, there is no dust, little molecular emission, and mostly neutral atomic gas for which emission line ratios directly probe the bulk elemental abundances. We visually summarize this series of processes in Fig. B.1.

The main source of uncertainty in these calculations comes from the charge exchange rate coefficients, which are accurate to within a factor of 2-4 (Kingdon \& Ferland 1996, 1999). The coefficients determine the amount of $\mathrm{H}^{+1}$ and, therefore, the electron density of the gas. In turn, the electron density impacts the level populations in the upper state of the transitions giving rise to the observed emission lines. The uncertainty in the rate coefficients leads to a systematic uncertainty of typically $20 \%$ on the synthetic line fluxes, as is discussed in Sect. 3.3.4 of Hazy, the Cloudy users' manual. We have included that uncertainty in the abundances listed in Table A.1. A secondary source of uncertainty in these calculations is the geometry of the emitting region. However, because we are fitting line ratios, we are not overly sensitive to the geometry of the emitting area, assuming that all of the lines originate in the same region.

An additional complication in the $\mathrm{N}$ abundance determination in less dense regions would normally be self-shielding of $\mathrm{N}_{2}$ against photodissociation, which is known to limit the conversion of $\mathrm{N}_{2}$ into atomic $\mathrm{N}$ or other $\mathrm{N}$-bearing molecules more efficiently than for $\mathrm{CO}$. However, as the excitation temperature increases, the self-shielding relative to other modes of shielding (e.g., by $\mathrm{H}, \mathrm{H}_{2}$, or $\mathrm{CO}$ ) becomes much less efficient (Heays et al. 2014), and a larger column of $\mathrm{N}_{2}$ would be required in order to self-shield. At the high temperatures and densities in our model $\left(T_{\mathrm{e}} \sim 6500 \mathrm{~K}, \log \left(n_{\mathrm{H}}\right) \sim 14.75 \mathrm{~cm}^{-3}\right)$, Cloudy indeed predicts that atomic $\mathrm{N}$ represents at least $99.8 \%$ of the total $\mathrm{N}$ budget. Critically, even if shielding were efficient at these temperatures, the fact that the UV field is generated locally by an $\mathrm{H}$ recombination would effectively prevent the formation of a sufficiently large column to shield, as the $\mathrm{H}+$ and $\mathrm{N}_{2}$ are intimately mixed. There is observational evidence, in any event, for $\mathrm{N}_{2}$ photodissociation already occurring further out in the disk. Hily-Blant et al. (2019) detected selective $\mathrm{N}_{2}$ photodissociation indirectly where the $\mathrm{N}_{2}$ snowline starts in the upper layers of the disk around $40 \mathrm{AU}$, through a variation in the ${ }^{15} \mathrm{~N} /{ }^{14} \mathrm{~N}$ ratio in ALMA observations of $\mathrm{HCN}$ isotopologs. And in our own data, we see the $1.04 \mu \mathrm{m}[\mathrm{N} \mathrm{I}]$ quadruplet at all three epochs; however, it is subtracted out of the epoch residual spectrum, suggesting that it originates further out in the disk rather than in the ring of denser material that we modeled moving toward the star in 2013.

As an independent check on the resulting hydrogen density, we ran CHIANTI models of line ratios taken between the $\mathrm{C}^{0}$ lines, as in McClure (2019), with additional line ratios between the $\mathrm{S}^{0}$ triplet. The range of electron densities and electron temperatures produced by these abundance- and geometry-independent measures for both $\mathrm{C}$ and $\mathrm{S}$, combined, are $n_{\mathrm{e}} \sim 2 \times 10^{10}-10^{12} \mathrm{~cm}^{-3}$ at $T_{\mathrm{e}} \sim 7500-5500 \mathrm{~K}$. The best-fitting Cloudy model has a value of $n_{\mathrm{e}} \sim 10^{11} \mathrm{~cm}^{-3}$ at $T_{\mathrm{e}} \sim 6500 \mathrm{~K}$, which is consistent with CHIANTI's predicted range.

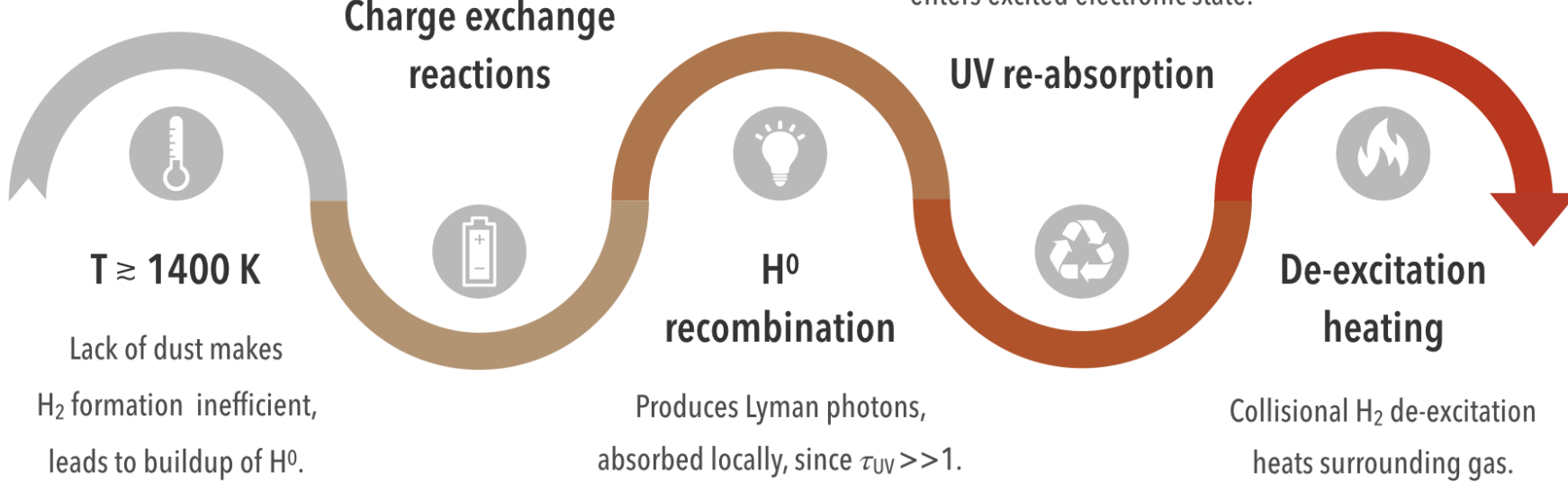

Fig. B.1. Flowchart of the inner disk physical processes leading to the high temperatures and domination of $\mathrm{H}$ over $\mathrm{H}_{2}$ and $\mathrm{C}$ over $\mathrm{CO}$ at the disk midplane, in contrast to the typical photon-dominated region physics seen in molecular clouds and the upper layers of the outer disk. 


\section{Appendix C: Carbon locking fractions}

As an independent check on the location where carbon is locked out of the accreting gas, we formulated a toy model, pictured in Fig. 1 of the main text. First, we divided the disk into three zones: Zone 1 (between the outer disk edge and the CO snowline), Zone 2 (between the $\mathrm{CO}$ snowline and inner rim of the dust), and Zone 3 (interior to the inner rim of the dust). Then we define the model as a parcel of constant density gas and dust that initially sits in the outer disk but is radially transported via accretion processes through the disk to the launching region for the stellar magnetospheric accretion columns. The parcel starts as carbon gas, $M_{\mathrm{g}, 0}$, with overall solar carbon abundances of $X_{\mathrm{g}, 0}=(\mathrm{C} / \mathrm{H})_{\odot}=2.69 \times 10^{-4}$ (Asplund et al. 2009), from which in Zone 1 solid carbon condenses out with a mass of

$M_{\mathrm{d}, 1}=M_{\mathrm{g}, 0}-M_{\mathrm{g}, 1}$,

leaving a depleted carbon gas mass of $M_{\mathrm{g}, 1}$ and a corresponding abundance of $X_{\mathrm{g}, 1}$ with respect to hydrogen. In the main text, we assume $X_{\mathrm{g}, 1}=6.4 \times 10^{-7}$ (Zhang et al. 2019).

The solid carbon component can be split into volatile ice, as $\mathrm{CO}$, and dust that sublimates at higher temperatures than $\mathrm{CO}$, with the fraction $f_{\mathrm{r}}$. The higher temperature carbon could be in any of the following materials: $\mathrm{CO}_{2}$ or other $\mathrm{C}$-rich ices, organic residues, amorphous carbon, or graphite. The grains are assumed to move radially along with the gas, unless they become decoupled from the accretion stream and locked in place in one of the zones. This locking could either be caused by the dust decoupling from the gas and entrapment of larger, millimeter-sized dust grains at pressure maxima or by the formation of bodies that are neither large enough to accrete with the gas nor radially drift. The model is agnostic to the exact locking mechanism. We do not consider complications from the decoupling of the gas and nonlocked dust due to radial drift; we assume that this dust moves with the gas.

We define a fraction, $f_{\mathrm{L}, 1}$, of the initial dust, $M_{\mathrm{d}, 1}$, that is locked in some manner in Zone 1, outside of the CO snowline, while the remaining dust exists in small, coupled grains that accrete with the gas across the CO snowline. The successfully transported dust is defined as:

$M_{\mathrm{d}, \mathrm{d}, 1}=\left(1-f_{\mathrm{L}, 1}\right) M_{\mathrm{d}, 1}$.

At the CO snowline, the volatile dust sublimates, releasing carbon back into the gas phase. Therefore, the new carbon gas mass is

$M_{\mathrm{g}, 2}=M_{\mathrm{g}, 1}+\left(1-f_{\mathrm{r}}\right) M_{\mathrm{d}, \mathrm{d}, 1}$,

which can be rewritten as

$M_{\mathrm{g}, 2}=M_{\mathrm{g}, 1}+\left(1-f_{\mathrm{r}}\right)\left(1-f_{\mathrm{L}, 1}\right)\left(M_{\mathrm{g}, 0}-M_{\mathrm{g}, 1}\right)$, and it corresponds to a carbon abundance of $X_{\mathrm{g}, 2}$ with respect to hydrogen. In the main text, we assume $X_{\mathrm{g}, 2}=2.5 \times 10^{-6}$ (Zhang et al. 2019). Solving Eq. (C.4) for the fraction of dust locked in Zone $1, f_{\mathrm{L}, 1}$, and rewriting the gas masses of each zone in terms of their observed $\mathrm{C} / \mathrm{H}$ abundances, $X_{\mathrm{g}, 1}$ and $X_{\mathrm{g}, 2}$, yields

$f_{\mathrm{L}, 1}=1-\frac{1}{1-f_{\mathrm{r}}} \cdot \frac{X_{\mathrm{g}, 2}-X_{\mathrm{g}, 1}}{X_{\mathrm{g}, 0}-X_{\mathrm{g}, 1}}$

assuming that no appreciable hydrogen mass is locked into the grains. The interior of the dust mass to the $\mathrm{CO}$ snowline is then the fraction of the transported solids with a higher sublimation temperature than $\mathrm{CO}$ :

$M_{\mathrm{d}, 2}=f_{\mathrm{r}} M_{\mathrm{d}, \mathrm{d}, 1}$.

A fraction of the dust mass in Zone $2, f_{\mathrm{L}, 2}$, is also eventually locked, either through the process of growth to large grains or to planetesimal sizes. Therefore, the mass of dust that is transported in the parcel across the inner dust rim is the dust mass in Zone 2 less the fraction of dust mass $M_{\mathrm{d}, 2}$ locked in Zone 2:

$M_{\mathrm{d}, \mathrm{d}, 2}=\left(1-f_{\mathrm{L}, 2}\right) M_{\mathrm{d}, 2}$.

The mass of the gas phase carbon interior to the innermost dust rim is then the carbon gas mass of Zone 2, plus the additional mass of higher temperature transported dust, all of which now sublimates:

$M_{\mathrm{g}, 3}=M_{\mathrm{g}, 2}+M_{\mathrm{d}, \mathrm{d}, 2}$.

The latter can be rewritten as

$M_{\mathrm{g}, 3}=M_{\mathrm{g}, 2}+\left(1-f_{\mathrm{L}, 2}\right) f_{\mathrm{r}}\left(1-f_{\mathrm{L}, 1}\right)\left(M_{\mathrm{g}, 0}-M_{\mathrm{g}, 1}\right)$.

Zone 3 corresponds to the carbon abundance found in this work, $X_{\mathrm{g}, 3}=3.4 \times 10^{-6}$. Solving Eq. (C.9) for the fraction of dust mass $M_{\mathrm{d}, 2}$ locked into Zone $2, f_{\mathrm{L}, 2}$, yields

$f_{\mathrm{L}, 2}=1-\frac{1}{\left(1-f_{\mathrm{L}, 1}\right) f_{\mathrm{r}}} \cdot \frac{X_{\mathrm{g}, 3}-X_{\mathrm{g}, 2}}{X_{\mathrm{g}, 0}-X_{\mathrm{g}, 1}}$.

This locked fraction, which is in terms of the Zone 2 dust mass, can be converted via the definition of $M_{\mathrm{d}, 2}$ into a locking fraction in terms of the initial dust mass, $M_{\mathrm{d}, 1}$. We cannot simultaneously constrain both the locking fraction and the higher temperature carbon fraction, but if we assume that the latter is equivalent to the refractory carbon fraction, then a reasonable value range is from $f_{\mathrm{r}}=0.5$ in the ISM to $f_{\mathrm{r}}=0.83$ in comet $67 \mathrm{P}$, as is discussed in the main text. Using this range of values and the three observed carbon abundances discussed above produces locking fractions outside (Zone 1) and inside (Zone 2) the CO snowline ranging from $90-99 \%$. 